\title{
COMPORTAMIENTO DE TEMPLOS COLONIALES EN EL SISMO DEL 7 DE SEPTIEMBRE DE 2017 EN CHIAPAS
}

\author{
Jorge A. Aguilar Carboney ${ }^{(1)}$, Raúl González Herrera ${ }^{(2)}$, \\ Vicente Guerrero Juárez ${ }^{(3)}$ y Manuel Jara Díaz ${ }^{(4)}$
}

\begin{abstract}
RESUMEN
En este artículo se presenta una revisión de edificios coloniales en Chiapas, México, dañados por el sismo del 7 de septiembre del $2017\left(\mathrm{M}_{\mathrm{w}}=8.2\right)$. Se hace una breve descripción de la estructuración de los edificios analizados, los daños observados y procesos de intervención a los que han sido sometidos, y se describe su comportamiento y causas de los daños provocados por el sismo del 7 de septiembre del 2017. Se presentan algunas reflexiones derivadas de la experiencia con los diferentes criterios de ingeniería estructural que se han aplicado a estas edificaciones históricas.
\end{abstract}

Palabras clave: edificios históricos; vulnerabilidad sísmica; comportamiento sísmico

\section{PERFORMANCE OF COLONIAL TEMPLES DURING THE EARTHQUAKE OF SEPTEMBER 7, 2017 IN CHIAPAS}

\begin{abstract}
This article presents a review of colonial buildings in the state of Chiapas, Mexico, damaged by the earthquake of September 7th, $2017\left(\mathrm{M}_{\mathrm{w}}=8.2\right)$. A brief description is given of the structure of the buildings analyzed, their damage and their intervention processes to which they have been subjected, as well as their performance and damages during the September $7^{\text {th }}, 2017$ earthquake are described. The experience of structural engineering applied on historical building restoration projects are discussed.
\end{abstract}

Keywords: historical buildings; seismic vulnerability; seismic performance

Artículo recibido el 29 de mayo de 2018 y aprobado para su publicación el 19 de junio de 2020. Se aceptarán comentarios y/o discusiones hasta cinco meses después de su publicación.

(1) Facultad de Ingeniería, Universidad Autónoma de Chiapas, Blvd. Belisario Domínguez km 1081 S/N, Tuxtla Gutiérrez, Chiapas, México, C.P. 29050.jaguilar@unach.mx , jcarboney@gmail.com

(2) Facultad de Ingeniería, Universidad de Ciencias y Artes de Chiapas, Tuxtla Gutiérrez, Chiapas, México. ingeraul@yahoo.com

(3) Restaurador independiente, Tuxtla Gutiérrez, Chiapas, México. vguerrerojz@hotmail.com

(4) Facultad de Ingeniería, Universidad Michoacana de San Nicolás de Hidalgo, Morelia, Michoacán, México. jarad10@gmail.com

DOI: $10.18867 /$ ris. 102.502 


\section{INTRODUCCIÓN}

\section{Características del evento sísmico}

El día 7 de septiembre de 2017, el Servicio Sismológico Nacional (SSN) reportó un sismo con magnitud $\mathrm{M}_{\mathrm{w}}=8.2$, que fue localizado en el Golfo de Tehuantepec, a $133 \mathrm{~km}$ al suroeste de Pijijiapan, Chiapas. El sismo, ocurrido a las 23:49:17 horas (04:49 UTM), fue sentido en el sur y centro de México. Las coordenadas del epicentro fueron $14.761^{\circ}$ latitud $\mathrm{N}$ y $-94.103^{\circ}$ longitud $\mathrm{W}$ y la profundidad fue de 45.9 km (SSN, 2017; Okuwaki y Yagi, 2017; Ye et al., 2017).

El mecanismo focal del sismo muestra una falla de tipo normal (rumbo $=311^{\circ}$, echado $=84.4^{\circ}$, desplazamiento $=-94.7^{\circ}$ ), la cual, es característica de un sismo intraplaca. En esta región, la placa de Cocos subduce (se desplaza) por debajo de la placa de Norteamérica y Caribe (SSN, 2017).

De acuerdo con el boletín de la Sociedad Mexicana de Ingeniería Sísmica (SMIS, 2017) en la zona cercana al epicentro el movimiento alcanzó aceleraciones de hasta $500 \mathrm{~cm} / \mathrm{s}^{2}$ aproximadamente en Santiago Niltepec, Oaxaca; de $230 \mathrm{~cm} / \mathrm{s}^{2}$ en Puerto Ángel, Oaxaca; Tuxtla Gutiérrez, Chiapas 70-200 cm/ $/ \mathrm{s}^{2}$ y de 108 $\mathrm{cm} / \mathrm{s}^{2}$ en Tapachula, Chiapas, de acuerdo con la Unidad de Ingeniería Sísmica del Instituto de Ingeniería de la Universidad Nacional Autónoma de México (UNAM).

Debido a la magnitud y profundidad del evento hubo miles de réplicas que generaron fatiga en las construcciones y provocaron daños a edificaciones ya de por sí dañadas, lo cual puso en relieve la necesidad de que la población, sobre todo la de las zonas de mayor daño en Oaxaca, Chiapas y Tabasco se proteja por los efectos de los daños acumulados. Las iglesias fueron cerradas y en algunos casos todavía no pueden abrir (SMIS, 2017).

\section{Experimentación y modelación matemática de edificaciones históricas}

El estado de Chiapas cuenta con un vasto patrimonio cultural de edificios históricos que fueron afectados por el sismo del 7 de septiembre del 2017, el cual presentó epicentro en la zona del Istmo de Tehuantepec en una región entre Chiapas y Oaxaca. En ambos estados se presentaron daños importantes en numerosos edificios históricos, principalmente en templos coloniales. En el estado de Chiapas algunas de estas edificaciones habían sido intervenidas estructuralmente en diferentes épocas, desde proyectos de rehabilitación menores y locales hasta procesos de refuerzo y restructuración integrales.

Este sismo de gran magnitud $\left(\mathrm{M}_{\mathrm{w}}=8.2\right)$, que provocó aceleraciones de terreno altas en la zona costa y central del Estado de Chiapas, sometió a prueba la eficiencia de las soluciones de ingeniería estructural utilizadas en la intervención de edificios históricos en esta zona del país.

De acuerdo con los datos del Sistema Integral de Protección Civil del Estado de Chiapas, los daños graves en edificios históricos y culturales en el estado por el sismo corresponden a los siguientes:

- 83 monumentos históricos en 35 municipios.

- 5 zonas arqueológicas.

- 13 museos.

- 1 monumento artístico.

No obstante, para entender este desastre es necesario recorrer el estado ya que fue eminentemente rural, se reportaron daños en 1,588 localidades de 97 municipios. Este documento se centra en el 
comportamiento estructural de cuatro templos coloniales que fueron sometidos a un proceso de rehabilitación estructural integral previo al sismo de 2017.

Los procesos de evaluación de la vulnerabilidad estructural de edificios históricos son muy complejos tal como se constata en las metodologías propuestas por Meli (1998); Simoes Dias Maio (2013); RiveraVargas y Arce-León (2014), entre otras. Uno de los elementos que dota de complejidad al proceso es la falta de experimentación en estos sistemas estructurales considerando los materiales y procesos constructivos que son variantes en cada época histórica en que fueron concebidos y desarrollados.

La falta de experimentación en monumentos históricos en México y particularmente en Chiapas según los autores de este manuscrito se debe fundamentalmente a tres factores:

- Lineamientos y restricciones generados por las entidades del gobierno que están a cargo de los edificios históricos ya que limitan con frecuencia las intervenciones con nuevas alternativas tecnológicas.

- Falta de recursos económicos y de personal capacitado e interesado en el tema de ingeniería estructural para la rehabilitación de edificios históricos en las diversas regiones del país.

- Prioridades de líneas de investigación de los diferentes fondos que promueven recursos para la investigación y generación de conocimiento.

Sin embargo, proyectos experimentales como los desarrollados en países europeos permiten tener datos suficientes para calibrar modelos matemáticos más sofisticados Elmenshawiab y Shriveb (2015); Peña-Mondragón (2014).

Los tipos de análisis estructural para construcciones históricas se pueden dividir en diferentes formas según Peña-Mondragón (2014):

- De acuerdo con su función: análisis para validación y calibración; análisis para la evaluación de la estructura.

- De acuerdo con el comportamiento del material: análisis elástico - lineal; análisis inelásticos.

- De acuerdo con el tipo de cargas: análisis dinámicos; análisis estáticos.

Así mismo, cabe recordar que, la evaluación de la seguridad estructural de un edificio histórico no debe estar basada únicamente en cálculos numéricos; sino también en las evidencias sobre el comportamiento del edificio ante eventos pasados, es decir, con base en su propia historia. Este análisis involucra diversos aspectos, además de un análisis numérico, propiamente dicho, como: análisis histórico, análisis de daños e intervenciones, análisis de la geometría y materiales, etc. (Peña-Mondragón, 2014).

\section{COMPORTAMIENTO DE EDIFICIOS HISTÓRICOS}

La restauración de edificios históricos en Chiapas se inició formalmente en 1974, cuando el gobierno del Estado solicitó apoyo a la Secretaría de Patrimonio Nacional para intervenir el conjunto conventual de Tecpatán, el cual se encontraba prácticamente en ruinas, y restaurar un retablo de la capilla del Rosario del templo de Santo Domingo en San Cristóbal de las Casas. Con estas dos obras se establece en Chiapas una delegación de la Dirección General de Conservación de Sitios y Monumentos del Patrimonio Cultural, formada por los arquitectos Javier Arredondo Vega y Vicente Guerrero Juárez, estos procesos de intervención se prolongaron durante tres años por la magnitud de las obras y la limitación de los recursos económicos. Sin embargo, se realiza una intervención mayor al templo de Santo Domingo donde se llevó acabo la impermeabilización de las bóvedas, e inyección de grietas. Por otro lado, en Tecpatán se 
implementó la primera etapa de un programa de restauración integral que se describe más adelante (Aguilar et al., 2002).

Posteriormente, en octubre de 1975 se presentaron una serie de sismos con epicentro en la zona central de Chiapas, con profundidades menores a los $5 \mathrm{~km}$ y magnitudes $\mathrm{M}_{\mathrm{b}}$ entre 4.7 y 5.1 (SMIS, 2000). Estos sismos provocaron severos daños en la ciudad de Chiapa de Corzo, muy cercana al epicentro, se reportaron un gran número de daños graves en estructuras de mampostería, aunque no se reportan víctimas, se provocó el éxodo masivo de la población por los efectos de estos temblores (Moguel, 1975; Moreno-Ceballo et al., 2019).

El convento de Santo Domingo de Chiapa de Corzo sufrió severos daños especialmente en la torre principal, también se afectó la fuente colonial de la plaza central. Esto generó la necesidad de un proyecto de intervención para restaurar estas edificaciones históricas, lo que permitió la continuidad de las tareas de restauración y consolidó las bases de un grupo de trabajo especializado en restauración, que se ha mantenido hasta estas fechas desarrollando diversas obras de intervención en Chiapas, cuyos trabajos se vieron evaluados tras la presencia del sismo del 7 de septiembre de 2017.

De acuerdo con los datos recabados por Aguilar et al. (2002); Alcocer et al. (1999); Jara (1999); Meli (1998); Meli (2003); Jara (2005) y Jara-Díaz et al. (2007), se concluye que los elementos estructurales más vulnerables de las iglesias son las partes altas de torres y campanarios, las fachadas y los apéndices. Sin embargo, desde el punto de vista de la gravedad para el sistema estructural, los elementos que más problemas presentan son las cúpulas, bóvedas y fachadas. Las torres y los campanarios son susceptibles de presentar daños particularmente en el caso de sismos. Se han observado contrafuertes exteriores con agrietamiento diagonal, señal de que han sido solicitados durante eventos telúricos.

\section{El exconvento de Santo Domingo en Chiapa de Corzo}

El exconvento de Santo Domingo en Chiapa de Corzo es una edificación del siglo XVI, a pesar de las reparaciones y modificaciones que ha sufrido durante su historia, es una de las edificaciones de esa época mejor conservadas en Chiapas. La estructura corresponde al convento colonial dominico típico, conformado por el templo estructurado por muros principales de carga y el área conventual, con un patio central cerrado por corredores con sistema de arcos. El material principal en los muros del edificio es el ladrillo, en la nave principal del templo se construyeron contrafuertes de poco espesor hacia el interior de la nave principal (figura 1). La torre del campanario en la zona oriente del templo, en la intersección del crucero y la capilla, es una estructura cuadrada robusta (Markman, 1993).

Los sismos de octubre de 1975 provocaron daños en el templo, especialmente en la zona del campanario. Para rehabilitar la estructura se desarrolló un proyecto de rehabilitación estructural dirigido por el Ing. Manuel González Flores, inicialmente se propuso un sistema de postensado vertical en la torre del campanario. El sistema de postensado consistió en colocar cables de acero anclados con elementos de concreto en la parte superior de la torre y se construyó una estructura adosada a la cimentación existente para sujetar los cables y postensarlo desde la base de la estructura. Posteriormente se decidió reforzar el edificio mediante el encamisado de las cuatro columnas del crucero con varilla adicional en la periferia y en la torre del campanario se adosaron cuatro columnas de concreto con trabes, formando marcos de concretos interiores. El edificio tuvo un comportamiento adecuado en sismos fuertes y de mediana intensidad previos a 2017, que no provocaron daños estructurales de consideración. 


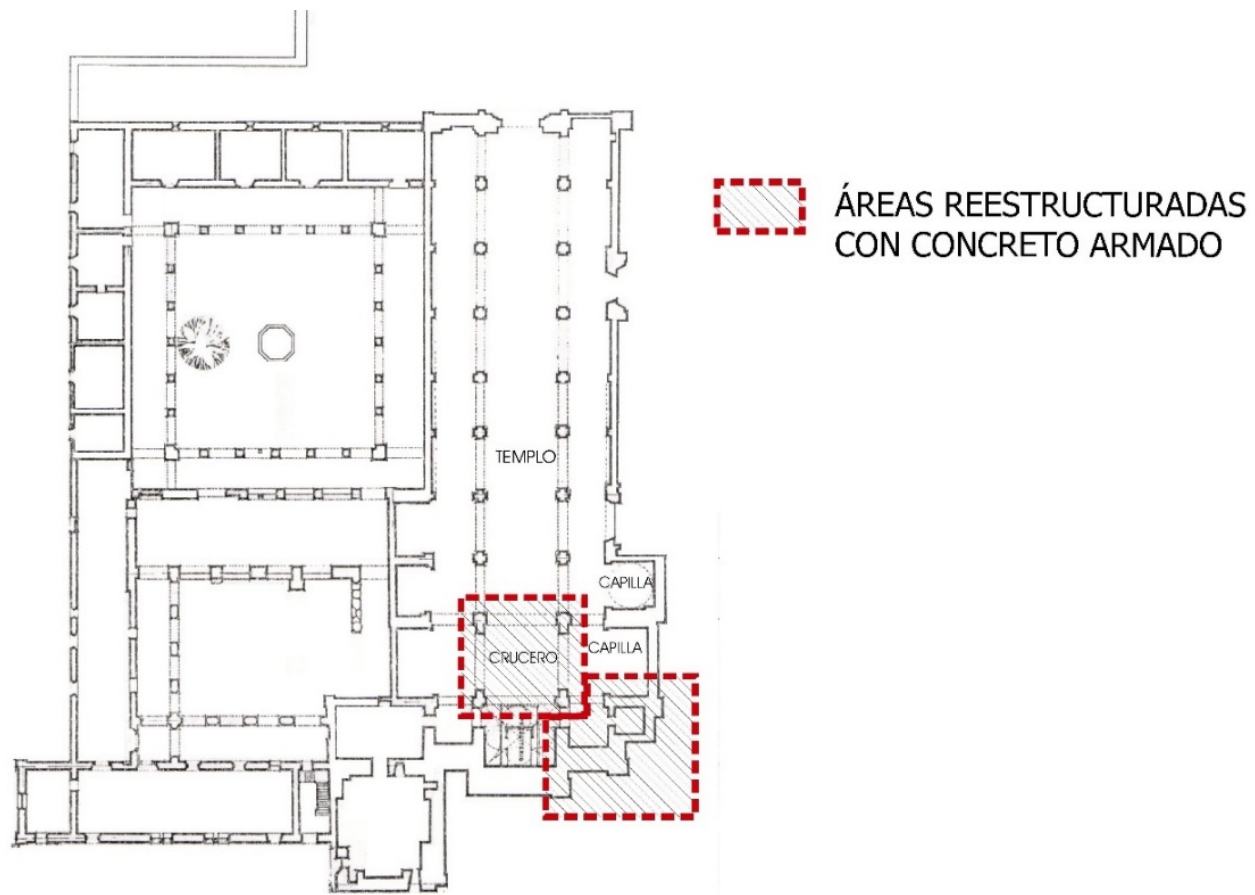

Figura 1. Planta del exconvento de Santo Domingo en Chiapa de Corzo

Los daños provocados por el sismo del 7 de septiembre de 2017 se concentraron en la zona del templo, la cúpula de la sacristía presentó severos agrietamientos y desprendimiento de material al interior, con riesgo de colapso, por lo que fue temporalmente apuntalada. El sismo generó movimientos diferenciales de los muros que provocaron el patrón de daños en las cúpulas mostrado en la figura 2. La parte alta de la fachada principal tuvo daños graves como se aprecia en la figura 3. Este daño fue extensivo en otras zonas del templo.

En el área del convento solamente se observaron grietas superficiales muy localizadas en uno de los muros. Es evidente el comportamiento diferente e independiente de la estructura del templo de la zona del convento.

En 2019 se definió un proyecto de rehabilitación estructural del templo que consiste principalmente en el uso de fibra de carbono y tensores de acero. La fibra de carbono se aplicará alrededor de los muros que soportan las cúpulas para funcionar como zunchos y proveer de mejor confinamiento, así como mayor capacidad a cortante de la estructura.

\section{El templo del Carmen en San Cristóbal de las Casas}

El templo del Carmen en San Cristóbal de las Casas es una edificación del siglo XVI, tiene una planta en "L" con una torre que sobresale en el extremo este de la nave principal (figura 4). Sus muros son parte de adobe y parte de piedra burda y ladrillo cubiertos con estuco, con un espesor promedio de $1.60 \mathrm{~m}$ (Markman, 1993). Este templo sufrió un incendio en marzo de 1995 que destruyó prácticamente todo el edificio, dejando en pie únicamente los muros de carga originales. 

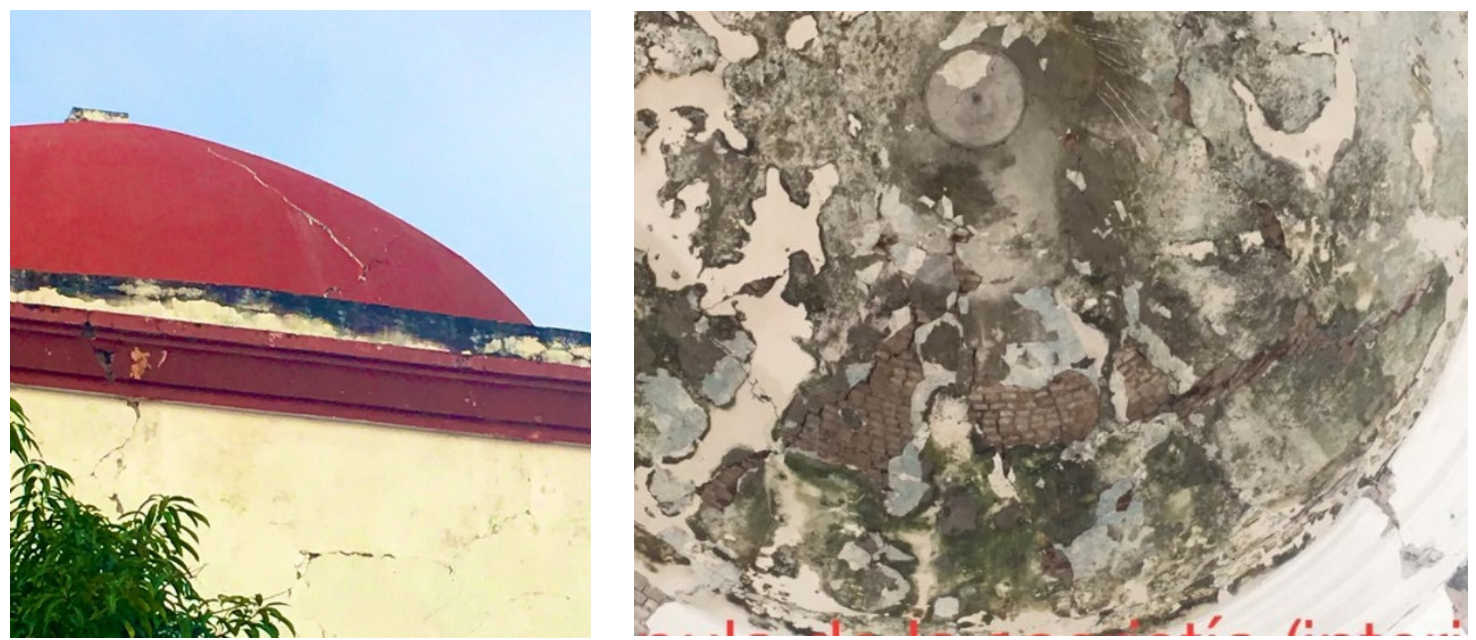

Figura 2. Daños en cúpulas

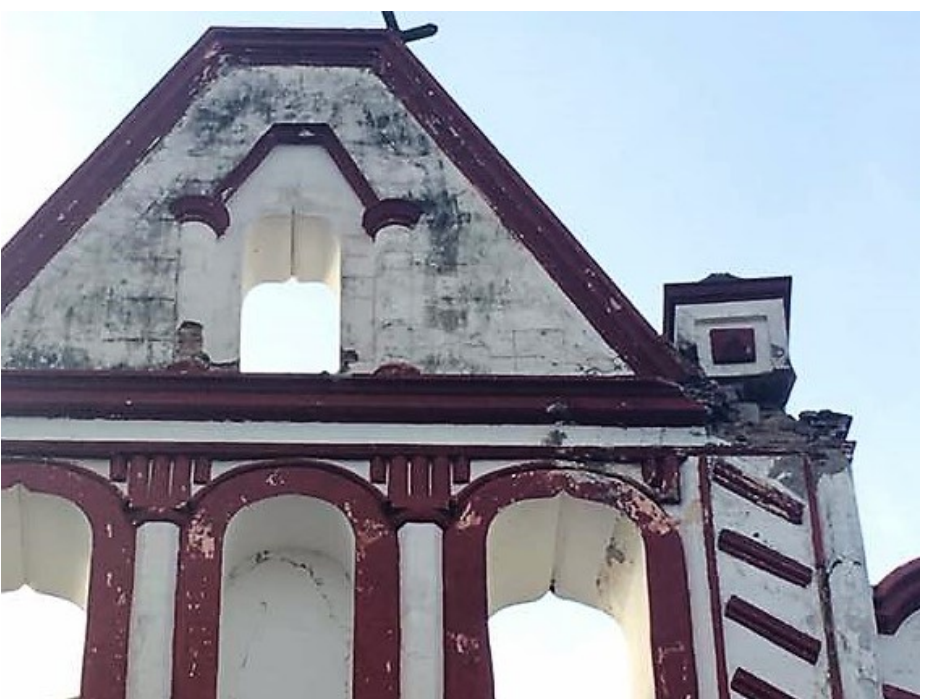

Figura 3. Agrietamientos en la parte alta de la fachada

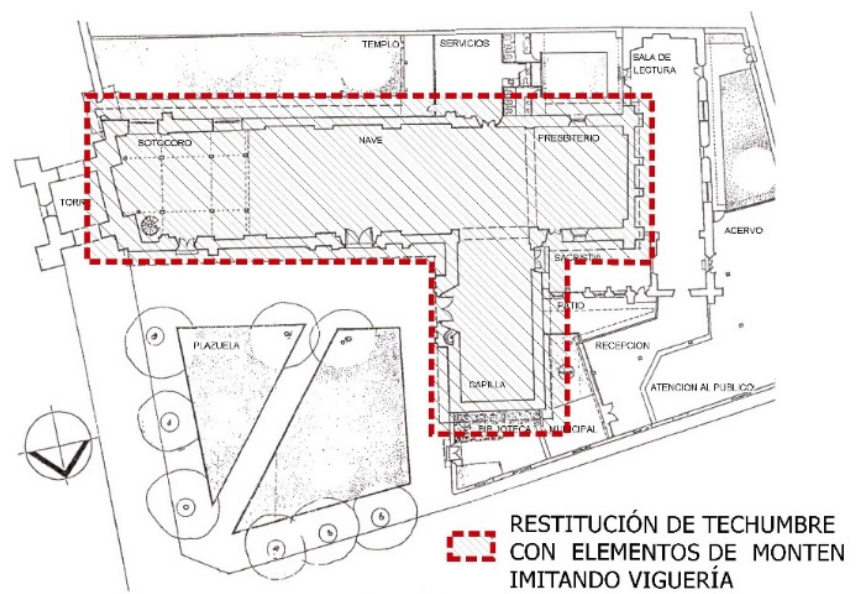

Figura 4. Planta del templo del Carmen 
Se implementó un proyecto de intervención integral del templo. Para la restauración de la techumbre se sustituyó la viguería de madera original con elementos metálicos de pared delgada. La estructura metálica se construyó con la misma geometría que la estructura de madera, con la intención de conservar el esquema estructural original y mejorar la rigidez de la techumbre. La estructura de metal quedó oculta por un artesón de madera como plafón del templo y por el tejado tradicional en la parte superior. En la figura 5 se presenta un esquema de la reparación realizada tras el incendio de la cubierta (Aguilar et al., 2002).

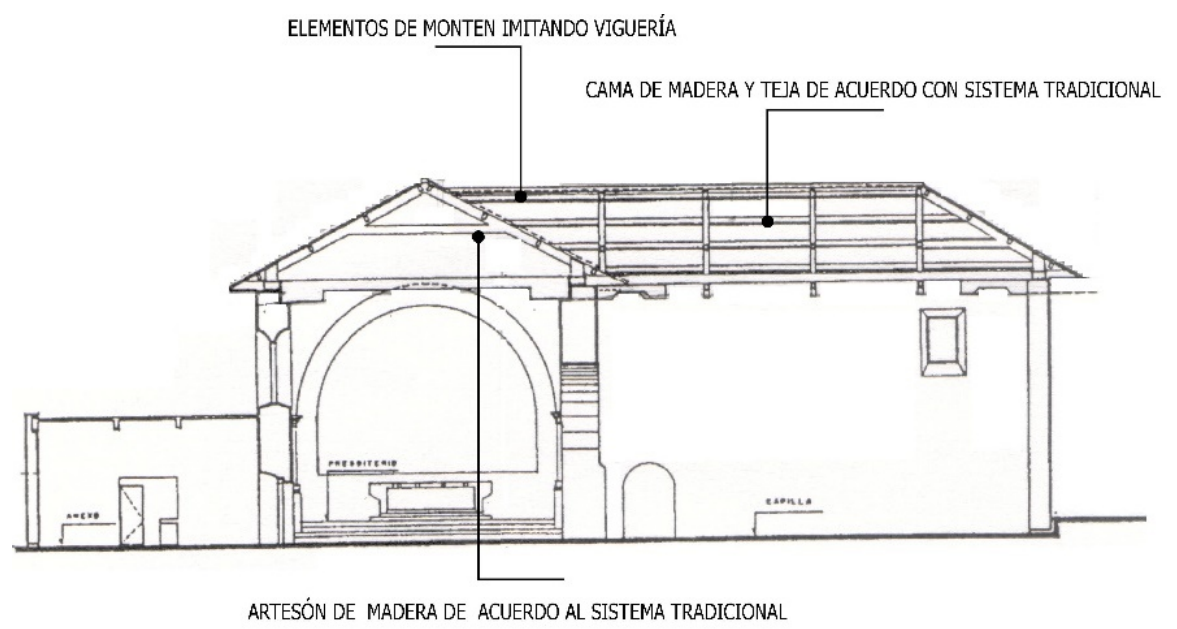

Figura 5. Restauración de la techumbre en el templo del Carmen

Este edificio se comportó adecuadamente durante el sismo del 7 de septiembre 2017, no presentó daños estructurales, solamente se observaron desprendimientos menores de acabados en la fachada, lo cual implica que la intervención que se realizó fue adecuada.

Las armaduras metálicas que conectan los muros laterales en el templo funcionaron como tensores que controlaron los desplazamientos diferenciales de estos muros, y además permitieron la estabilidad de la techumbre y mejoraron la integridad estructural.

\section{El templo de Santa Lucía en San Cristóbal de las Casas}

El templo de Santa Lucía es una edificación del siglo XIX, la nave principal tiene tres capillas adosadas y dos torres en el frente (figura 6). Este templo sufrió graves daños en todo el edificio en el sismo del 20 de octubre de 1995, con epicentro en la zona central de Chiapas (González et al., 2017).

La fachada principal actual, que incluye los campanarios al oriente de este edificio, fue construida en una etapa posterior al resto del templo y su cimentación es independiente, anexa a la del templo. En el sismo de 1995, la estructura de esta fachada se inclinó hacia el frente con el peligro evidente de desplomarse y se presentaron agrietamientos severos en varios de los muros interiores, por lo que se implementó un proyecto de rehabilitación integral. Se estableció un programa de restitución de materiales e inyección de grietas con morteros expansivos en los muros dañados (figura 7). Este procedimiento se realizó limpiando la grieta con aire a presión y posteriormente sellándola superficialmente, dejando boquillas para la inyección del mortero (Aguilar et al., 2002). 


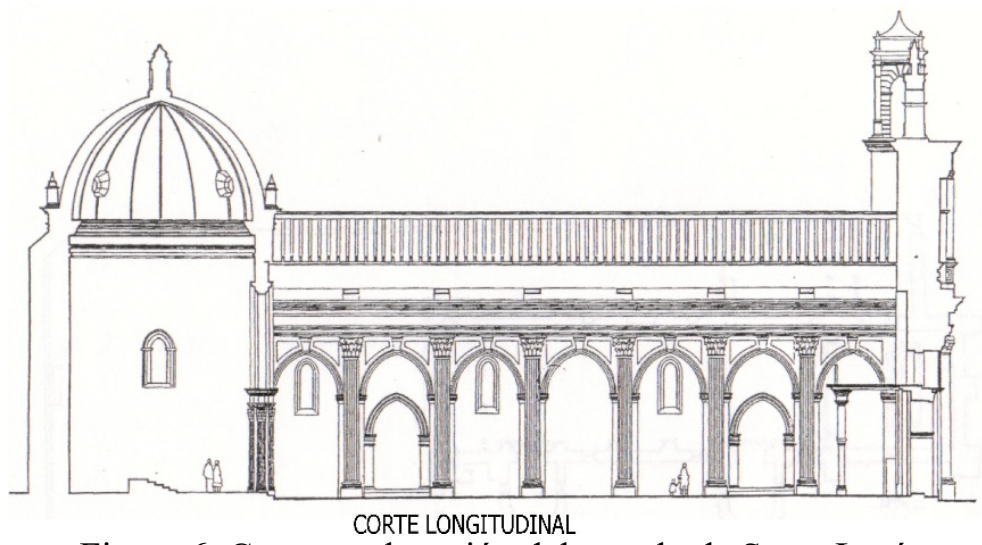

Figura 6. Corte en elevación del templo de Santa Lucía

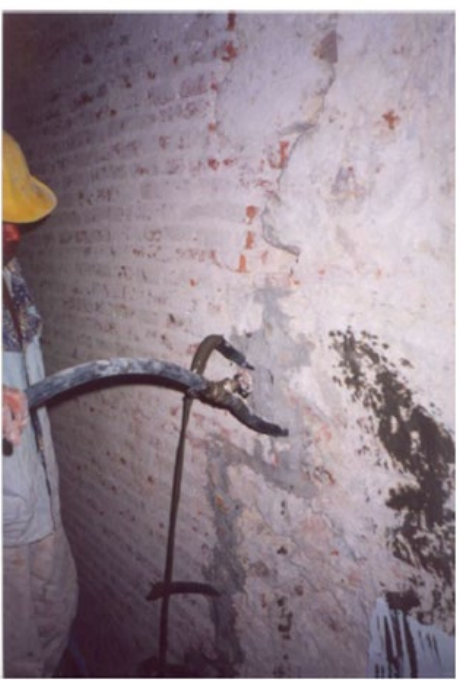

Figura 7. Inyección de grietas en muros

Para corregir el desplome de la fachada se decidió demoler y reconstruir su estructura original anclándola a los muros longitudinales, perpendiculares a la fachada, mediante la adición de una cadena de concreto reforzado en todo el rededor de la corona de los muros. El anclaje entre la cadena y la estructura de la fachada se realizó mediante elementos formados por placas de acero que quedaron ahogados en los muros, como se muestra en la figura 8.

Los elementos de madera originales de la techumbre se conservaron en su sitio, pero fueron reforzados en sus extremos con placas de acero para conectarlos al sistema de refuerzo estructural (figura 9). Se adicionaron estructuras metálicas entre los ejes de las estructuras de madera originales, para tomar las cargas de la techumbre. Los nuevos elementos se colocaron entre la viguería de madera existente para la estructura original. En la figura 10 se muestran detalles de la conexión entre la estructura de refuerzo y las estructuras de madera originales, así como el conjunto de estructuras metálicas ya colocadas sobre los muros. 

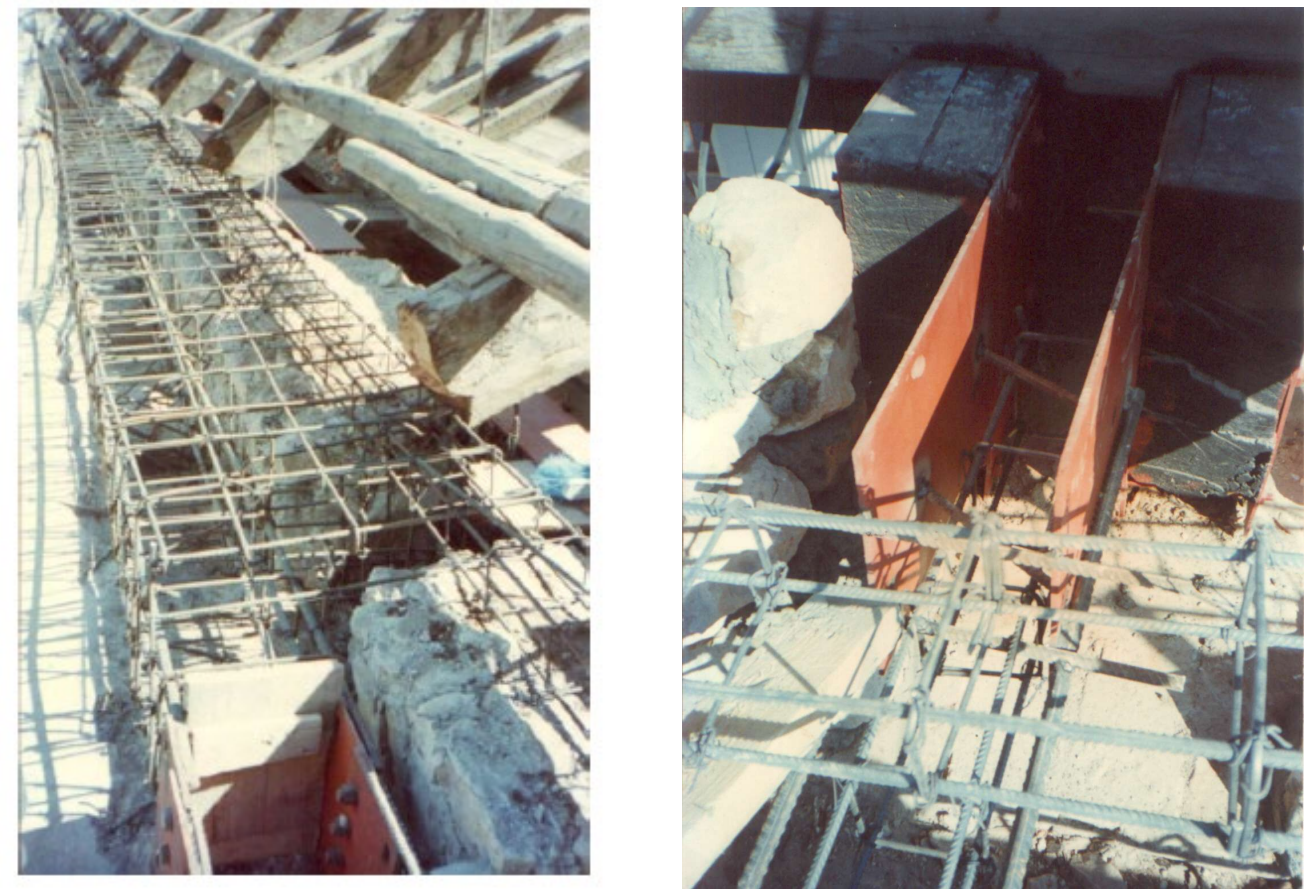

Figura 8. Cadena de cerramiento en la corona de muros principales
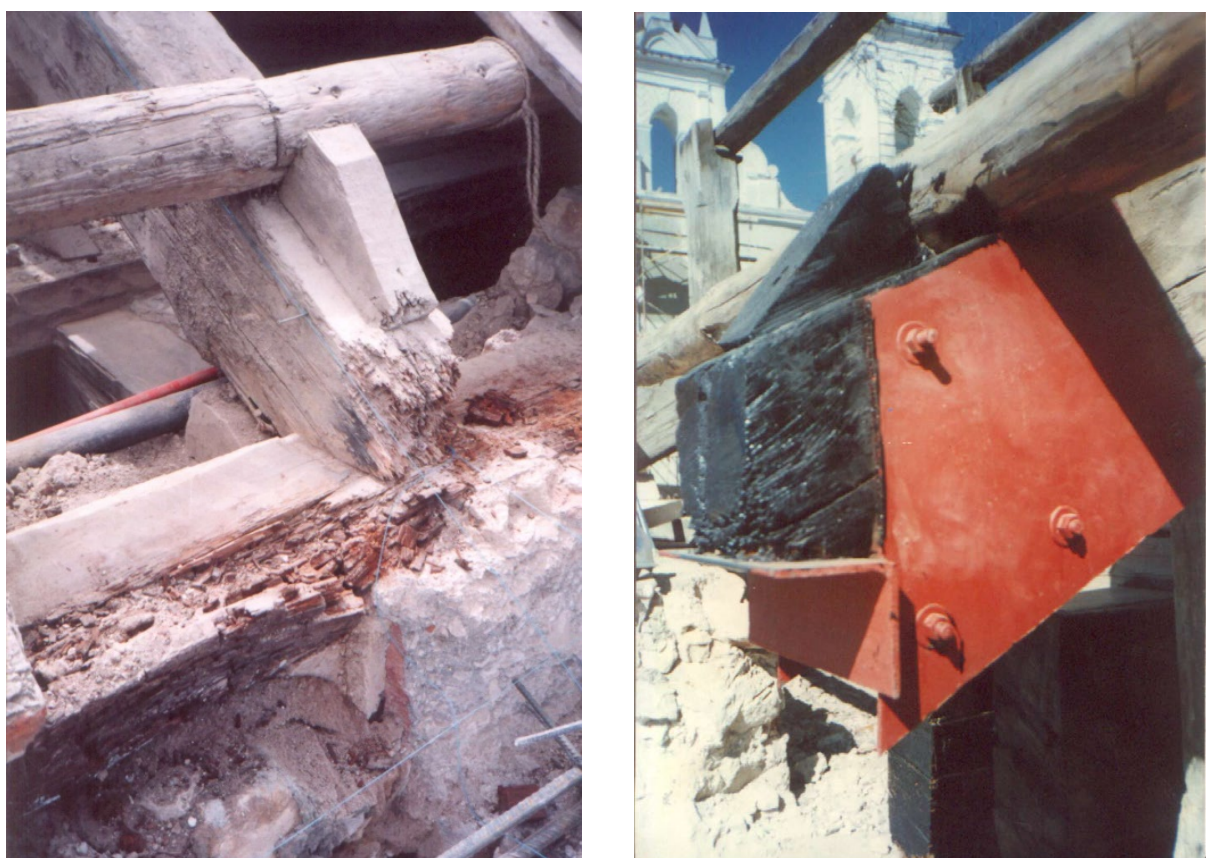

Figura 9. Viguería deteriorada en techumbre y detalle de restauración de la techumbre con acero 

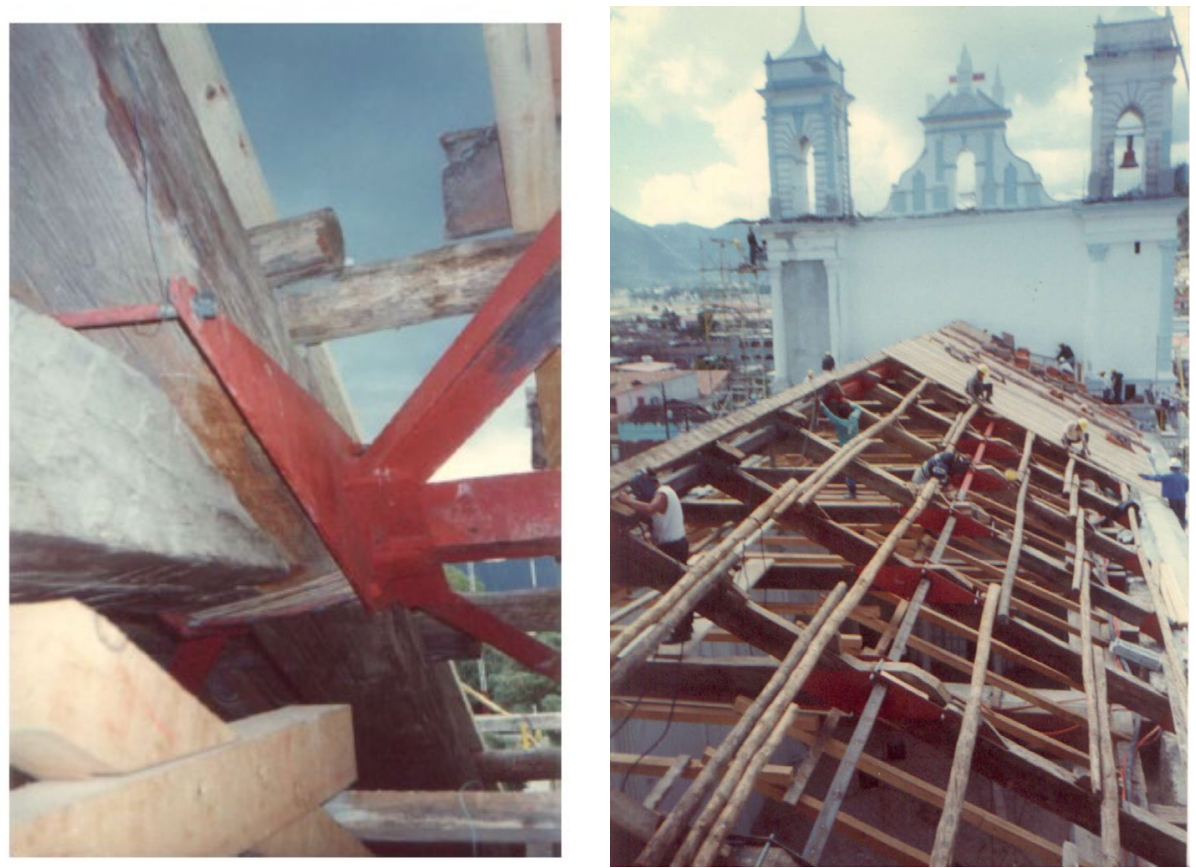

Figura 10. Rehabilitación de techumbre con estructura metálica

La estructura de la fachada que resultó muy dañada en el sismo de 1995 fue prácticamente removida en su totalidad, las columnas originales se demolieron junto con su cimentación. Estas columnas se reconstruyeron de concreto armado con una cimentación de mampostería adosada a la cimentación de la nave principal (figura 11). Tanto en la fachada del templo como en los muros interiores, adicional a la inyección de grietas, los agrietamientos más severos se resanaron con mortero con estabilizador de volumen y se sustituyeron piezas dañadas.

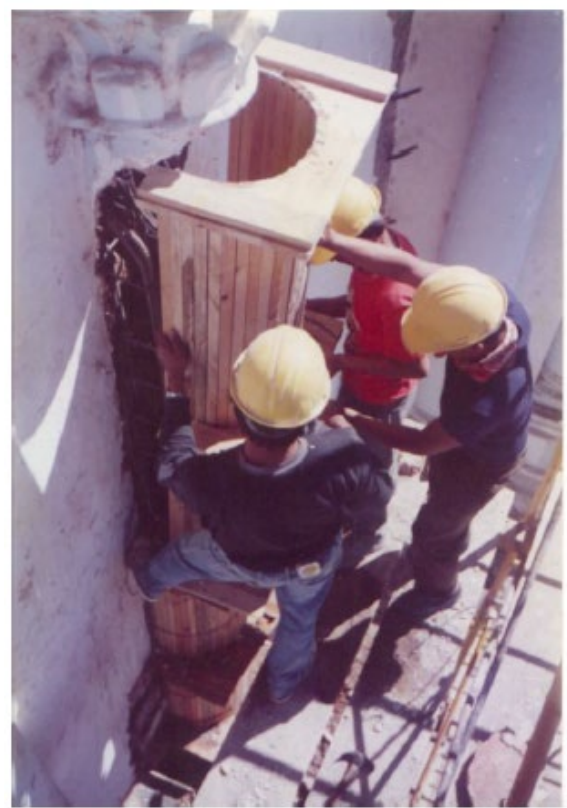

Figura 11. Restauración de las columnas de la fachada 
En el sismo del 7 de septiembre de 2017 la nave principal se comportó adecuadamente, sin embargo. la fachada sufrió daños graves, principalmente en campanarios y espadaña, los cuales colapsaron debido a desplazamientos considerables, como se muestra en la figura 12. También se presentaron daños en arcos interiores en la nave del templo (figura 12).

Las torres de campanarios y la espadaña funcionaron como apéndices muy frágiles sobre una estructura rígida. La fachada anclada a los muros longitudinales por la intervención estructural previa no tuvo daños de consideración, por lo que se observó un buen comportamiento del sistema, sin embargo, en el análisis no se consideró el comportamiento de los campanarios y espadaña como apéndices.
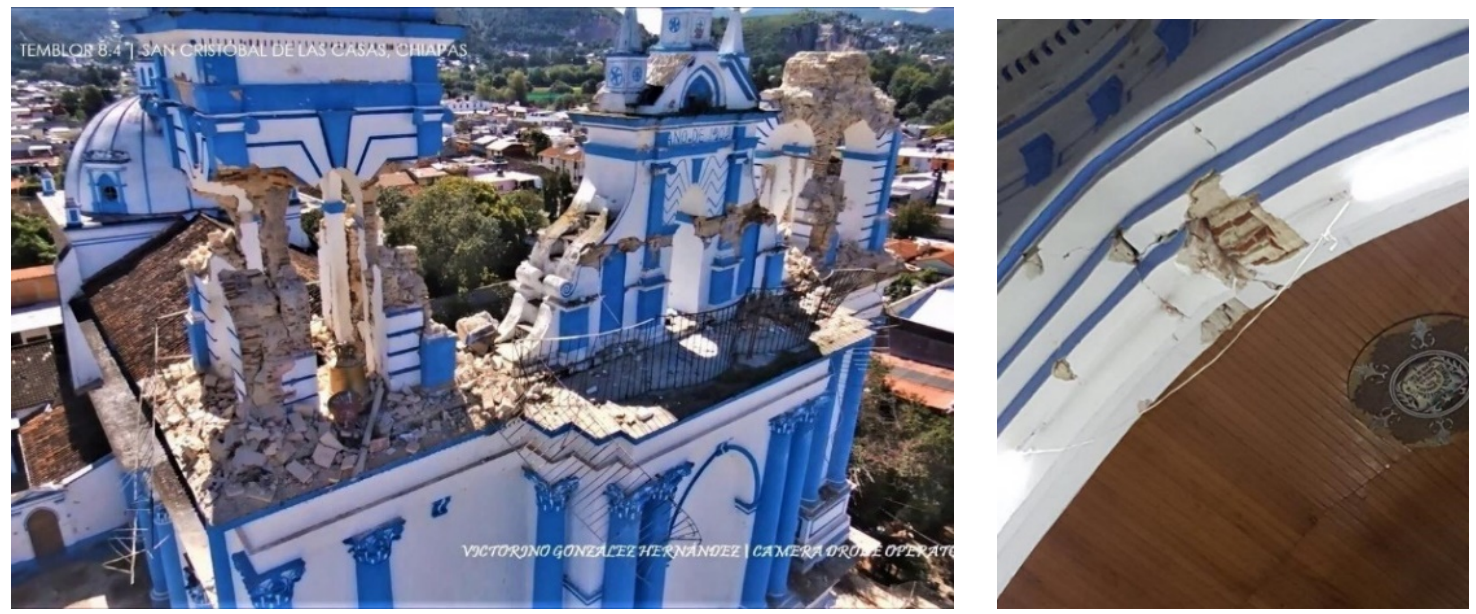

Figura 12. Daños en arcos interiores y fachada

\section{El templo de Santo Domingo en Tecpatán}

El templo y el convento dominico de Tecpatán, construido en el siglo XVI, es un ejemplo del carácter de los misioneros dominicos que frecuentemente reflejaban en sus construcciones una esperanza de desarrollo futuro, más que satisfacer una necesidad inmediata, concretando impresionantes edificaciones (Markman, 1993; Guzmán-Monrroy, 2005; Parrilla-Albuerne, 2015).

El conjunto conventual ha recibido varias intervenciones especializadas parciales desde 1974. En la actualidad está ocupado en algunas áreas restauradas. El grado de deterioro es variable, algunas zonas del templo y del convento están en ruinas. El templo conserva la fachada y muros de la nave principal, pero el sistema de techumbre está destruido. La torre principal de la fachada muestra una configuración muy robusta, lo cual puede inferir que sufrió daños en sismos históricos y fue rehabilitada confinándola con material similar al original, una alternativa utilizada en otros templos. Esto lo hace muy robusta con relación a la arquitectura para este tipo de edificación colonial (figura 13). Las intervenciones estructurales más recientes están enfocadas a la rehabilitación de la estructura del convento utilizando diferentes soluciones.

En los últimos años, se desarrolló un programa muy ambicioso de intervención del templo y el convento que pretende una restauración integral. Se realizaron modelos con elemento finito de la estructura para analizar su comportamiento, además se propuso realizar la instrumentación permanente del edificio. El proyecto de intervención pretende conservar en lo posible la configuración estructural original de la edificación. 


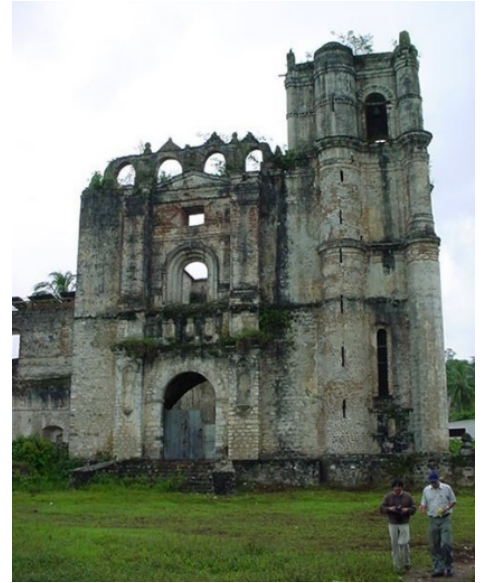

Figura 13. Templo de Santo Domingo en Tecpatán

En la última restauración del cuerpo de dos niveles de acceso al convento se implementó un sistema de tensores inmersos en las bóvedas que conectan las columnas a la altura de los riñones de la arquería. Los elementos tensores consisten en un sistema de barras de acero sólidas dentro de un elemento tubular que permite trabajar tanto en tensión como en compresión. Este sistema ha trabajado de manera eficiente ya que no se han detectado daños en sismos importantes (figura 14).

Los tensores están encamisados con tubos de acero, se conectan a las columnas y muros de mampostería mediante placas de acero. La función del sistema de tensores es restringir el desplazamiento de las columnas existentes y de los apoyos de las bóvedas interiores, permitiendo una mejor liga estructural entre los arcos interiores y los muros perimetrales. Este procedimiento ha demostrado ser muy efectivo para limitar los desplazamientos laterales de muros y columnas, el agrietamiento de los techos y evitar su colapso.
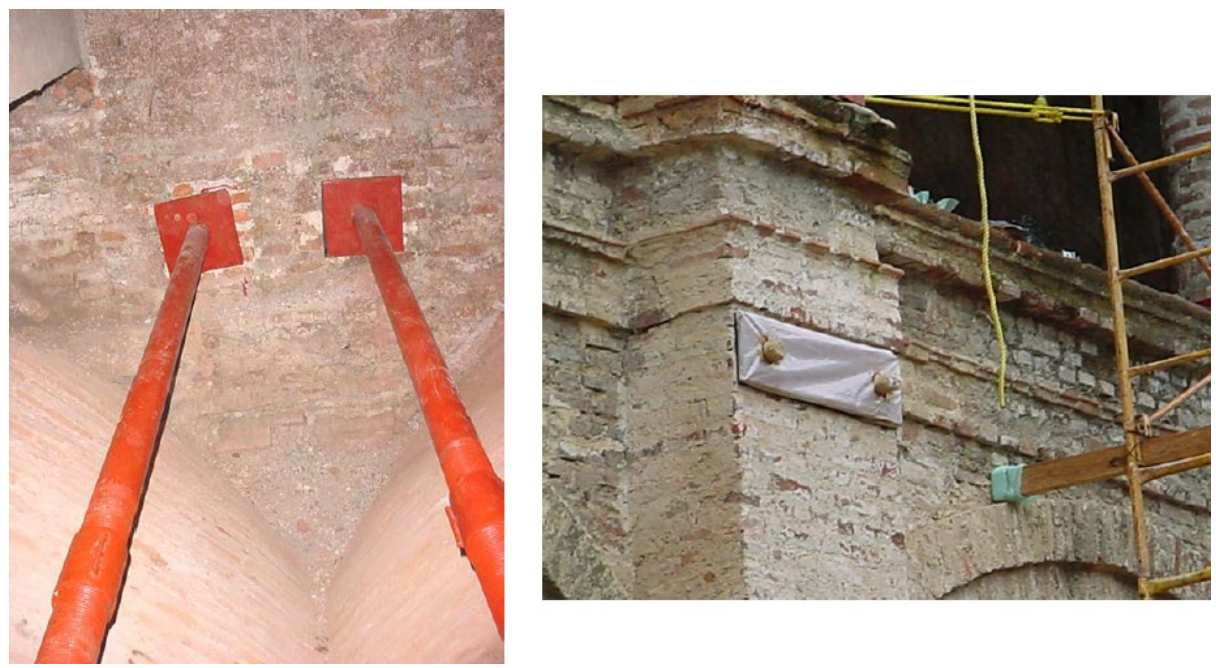

Figura 14. Tensores con camisa de acero y placa de conexión

Para restaurar las bóvedas, se utilizaron procesos constructivos tradicionales; se retiró el relleno de las bóvedas y se colocó una losa de concreto reforzado soportada en los muros y arcos paralelos, sustituyendo a una losa similar usada en una intervención anterior de esta zona, por lo que se disminuyó la magnitud de la carga vertical. 
Para la techumbre se implantó un sistema de armaduras de madera siguiendo la configuración estructural tradicional utilizada originalmente en la construcción de este tipo de techos y que aún es utilizada en la región. Se adicionaron tensores de acero entre cada armadura paralelos a la cuerda inferior, para ligar los muros en el entrepiso superior. Esta solución se usó también para el rescate de la techumbre de la crujía sur, colindante con uno de los muros principales del templo.

La segunda etapa de intervención consistió en la rehabilitación de la crujía norte, la cual tenía la arquería de mampostería muy destruida, se restituyeron los arcos, bóvedas y columnas siguiendo el sistema y materiales originales de la construcción, y aplicando el sistema de tensores utilizado en el techo para la crujía oriente (Jara-Díaz et al., 2007).

Durante el sismo del 7 de septiembre del 2017, toda el área que ha sido intervenida hasta la fecha se comportó de manera adecuada, no se detectaron daños, salvo fisuras aisladas en zonas previamente dañadas que hace falta atender (figura 15). Esto implica un buen comportamiento de los sistemas de refuerzo estructural aplicados a la edificación.

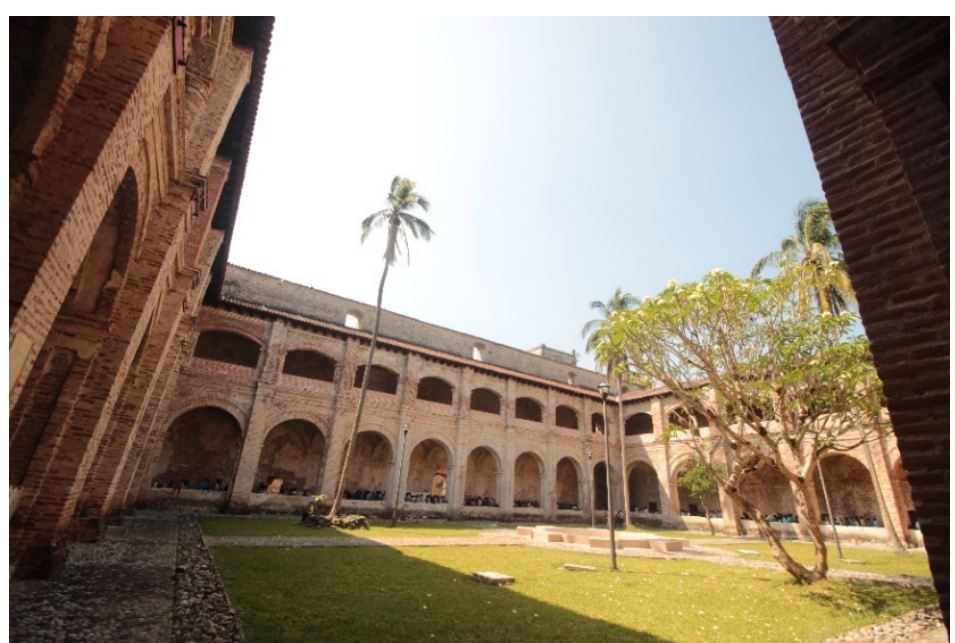

Figura 15. Interior patio principal del exconvento de Tecpatán

\section{CONCLUSIONES}

El sismo del 7 de septiembre de 2017 afectó numerosas construcciones coloniales en la zona sur de México, es importante rescatar la experiencia de los proyectos de intervención estructural implementados con diferentes alcances y en diferentes momentos. El comportamiento de estos edificios históricos ante un sismo de alta intensidad permite mejorar las estrategias para incrementar la seguridad y preservación del acervo cultural, un reto permanente y complicado para la ingeniería estructural moderna. En este artículo se presentaron algunos casos representativos de la problemática, pero existen numerosos edificios históricos en la zona que nunca han tenido una intervención que mejore sus condiciones de seguridad.

Las técnicas de intervención estructural que se han empleado para rehabilitación han tenido un efecto positivo en la estructuración y contribuyen a una mejor respuesta sísmica de las construcciones históricas. El proyecto de rehabilitación debe analizar el sistema estructural de manera integral, considerando la interacción entre los diferentes cuerpos que lo componen, pero así mismo identificar el comportamiento particular de cada componente para verificar su estabilidad local. Esquemas de refuerzo para la techumbre 
por medio de elementos metálicos ocultos por el artesón original, como el que se describe en este artículo, mostraron su eficiencia para mejorar la participación en conjunto de los muros y arcos.

La normatividad estructural no considera edificaciones de este tipo, por lo que frecuentemente las características de los materiales utilizados en el diseño de los proyectos de rehabilitación son definidas por criterios no homogéneos basados en la experiencia empírica. Es fundamental promover la investigación sobre el comportamiento estructural de estos edificios y las características de los materiales tradicionales utilizados para su construcción.

La experiencia que nos aporta el sismo de 2017 sobre el comportamiento de estructuras históricas refleja diversos problemas de vulnerabilidad recurrente que deben considerarse cuando se define un proyecto de rehabilitación. En los templos es evidente que las torres de los campanarios y los pináculos en la parte más alta se comportan como apéndices muy vulnerables a los desplazamientos en un evento sísmico, debe considerarse su refuerzo que mejore su comportamiento. Otro aspecto importante es el control de desplazamientos en los apoyos de bóvedas y arcos por la fragilidad de estos elementos.

Es importante establecer un programa de monitoreo permanente de estos templos para conocer mejor su comportamiento ante sismos severos. Es muy importante la investigación de las propiedades de los materiales, los cuales son muy variables de acuerdo con la zona donde se construyó, esta información es primordial para modelar con mayor precisión las estructuras y definir el sistema de rehabilitación más adecuado. Es también fundamental que todo proyecto de ingeniería estructural para rehabilitar un edificio histórico inicie con la investigación de las diferentes intervenciones a que se le ha sometido durante su historia y que han alterado su estructuración original.

Debe procurase incentivar la interacción de los arquitectos restauradores y los ingenieros estructuristas para entender mejor la problemática inmersa en la restauración de los edificios históricos. El reto fundamental es conciliar la ingeniería estructural moderna y el respeto histórico a la arquitectura colonial.

Finalmente, el sismo del 7 de septiembre de 2017 nos enseñó que pese a la complejidad de las intervenciones en este tipo de estructuras y a la falta de experimentación y normativa, cuando la intervención se hace de una manera multidisciplinaria y con criterio estructural adecuado se mejora el comportamiento de los edificios.

\section{REFERENCIAS}

Alcocer, S M, G Aguilar, L Flores, L Durán, O López-Bátiz, M A Pacheco, C M Uribe y M Mendoza (1999), "El sismo de Tehuacán del 15 de junio de 1999", Centro Nacional de Prevención de Desastres, Informe IEG/01/99, México.

Aguilar-Carboney, J A, V Guerrero y A De la Cruz (2002). "Restauración de Edificios Históricos en Chiapas". XIII Congreso Nacional de Ingeniería Estructural, Puebla, Puebla, México.

Elmenshawi, A y N Shrive (2015), "Assessment of Multi-Wythe Stone Masonry Subjected to Seismic Hazards", Journal of Earthquake Engineering, 19:1, 85-106, DOI: 10.1080/13632469.2014.940631

García-Moreno, L M (2017), Comunicación personal con el secretario de protección civil. Tuxtla Gutiérrez, Chiapas. 
González-Herrera R, A Tena-Colunga, J C Mora-Chaparro, M A Borraz-Santiago, J A Aguilar-Carboney, R Cruz-Díaz, J A Figueroa-Gallegos, J A Chan-Chim, M S Ramírez-Centeno, R Vera-Noguez y C Narcía-López (2017), "El sismo de Villaflores, Chiapas, sus realidades y consecuencias". 397 p. ISBN 978-607-8573-13-4. Universidad Autónoma de Chiapas, Colección Letras sin papel, Tuxtla Gutiérrez, Chiapas, México. Consultado como ebook en:

http://www.espacioimasd.unach.mx/libro/num15/El_sismo_de Villaflores_Chiapas sus realidades y_consecuencias.php

Guzmán-Monrroy, V (2005), “Santo Domingo de Tecpatán, Chiapas... un pueblo... un convento”. Boletín de monumentos históricos, Tercera época número 4 mayo-agosto.

Jara-Díaz M, J M Jara-Guerrero, J A Aguilar-Carboney y H Varum (2007), "Rehabilitación de iglesias dañadas en México, dos casos de estudio". V Seminario de Arquitectura de Terra en Portugal. Consultado en línea en: https://ria.ua.pt/bitstream/10773/8409/1/D_005.pdf

Jara, J M, R Rojas y B A Olmos (2005), “Comportamiento de edificaciones durante el temblor de Colima del 21 de enero de 2003”, Ciencia Nicolaita, No. 41, p. 209-224, Morelia, Michoacán, agosto, 2005.

Jara M (1999), "Informe sobre el sismo de Tehuacán del 15 de junio de 1999", Reporte interno de la Facultad de Ingeniería Civil, Universidad Michoacana de San Nicolás de Hidalgo, Morelia, Michoacán, México.

Markman, S D (1993), “Arquitectura y urbanización en el Chiapas Colonial”, Serie Científica Chiapas No. 5, Consejo Estatal de Fomento a la Ingeniería y Difusión de la Cultura, Tuxtla Gutiérrez, Chiapas, México, mayo, 604 pp.

Meli, R (1998), Ingeniería estructural de los edificios históricos, Fundación ICA, AC, México.

Meli, R (2003), "Patologías en edificios históricos dañados por sismo". VII Congreso Latinoamericano de Patología de la Construcción y IX Congreso de Control de Calidad en la Construcción, Volumen 1, Control de Calidad, p. 101-114, Mérida Yucatán, México.

Moguel, G (1975), Periódico La Tribuna, No. 5885, Tuxtla Gutiérrez, Chiapas, México. octubre 7.

Moreno-Ceballo R, R González-Herrera, J A Paz-Tenorio, J A Aguilar-Carboney y C U Del Carpio-Penagos (2019), "Effects of Sediment Thickness upon Seismic Amplification in the Urban Area of Chiapa de Corzo, Chiapas, Mexico". Earth Sciences Research Journal, Vol 23, No. 2, 111-117. DOI: $\underline{10.15446 / \text { esri.v23n2.72623 }}$

Okuwaki, R y Y Yagi (2017), "Rupture process during the Mw 8.12017 Chiapas, Mexico earthquake: shallow intraplate normal faulting by slab bending", Geophysical Research Letters, 44, 11,81611,823. DOI: $10.1002 / 2017$ GL075956

Parrilla-Albuerne, A M (2015), "El antiguo convento de Santo Domingo en Tecpatán, Chiapas, México". Conaculta, UNICACH, 343 pp.

Peña-Mondragón, F (2014), "Estrategia Integral de Análisis Complementarios para la Conservación del Patrimonio Arquitectónico", ler Simposio Nacional de Ingeniería Estructural de Construcciones Históricas, Guanajuato, México. 
Rivera-Vargas, D y C Arce-León (2014). "Simplified Assessment of the Structural Safety of Historical Constructions". SAHC2014 - 9th International Conference on Structural Analysis of Historical Constructions, F Peña y M Chávez (eds.), Mexico City, Mexico, 14-17 October 2014.

Simoes Dias Maio, R A (2013), "Seismic vulnerability assessment of old building aggregates". Tesis de Maestría en Ingeniería Civil, Universidad de Aveiro, Portugal, 149 pp.

Servicio Sismológico Nacional (SSN) (2017). "Reporte especial del sismo del 7 de septiembre de 2017", consultado en línea el 03/04/2018: http://www.ssn.unam.mx/sismicidad/reportesespeciales/2017/SSNMX rep_esp_20170907_Tehuantepec_M82.pdf

Sociedad Mexicana de Ingeniería Sísmica (SMIS) (2000), Base Mexicana de Datos de Sismos Fuertes $(B M D S F)$. Disco Compacto, Volumen 2, México, D.F.

Sociedad Mexicana de Ingeniería Sísmica (SMIS) (2017), Boletín Sismos Recientes. Descargado en línea en: www.smis.org.mx

Ye, L, T Lay, Y Bai, K F Cheung y H Kanamori (2017), “The 2017 Mw 8.2 Chiapas, Mexico, earthquake: energetic slab detachment". Geophysical Research Letters, 44, 11,824-11,832. DOI: $\underline{10.1002 / 2017 G L 076085}$ 